\title{
Keith DiPetrillo (ed): “Cardiovascular Genomics: Methods and Protocols". Springer Protocols-Methods in Molecular Biology
}

\author{
Vol 573, Humana Press, 2009, Hard cover, pages 350, ISBN 978-1-60761-246-9, \\ ISSN 1064-3745, DOI 10.1007/978-1-60761-247-6
}

\author{
Dhavendra Kumar
}

Published online: 3 March 2010

(C) Springer-Verlag 2010

This book is a collection of articles edited by Dr Keith DiPetrillo and published by Humana Press in collaboration with Springer Protocols as part of the Methods in Molecular Biology series. The title of the book is probably misleading. The preface directs to complex cardiovascular phenotypes of atherosclerosis, stroke and kidney damage as the leading causes of cardiovascular mortality. There is no reference to other monogenic and polygenic cardiovascular phenotypes that are collectively significant for cardiovascular morbidity and mortality. The aim of the book appears to provide a practical guide on a number of laboratory-based molecular methods that are likely to be useful in carrying out human and animal experiments to generate data and information for developing molecular diagnostic and therapeutic strategies for complex cardiovascular conditions.

The contents of the book largely cover generic and commonly employed laboratory methods in any modern cell and molecular biology research laboratory. Although the book specifically refers to the cardiovascular genomics, it is mostly non-specific and there is limited reference to cardiovascular genetics or genomics. However, chapters 1-8 focus on atherosclerosis, ischemic heart disease and hypertension. The remaining chapters (9-18) offer practical guide on procedures and statistical methods that are mandatory to any investigator in genomics and molecular biology. Most chapters describe invasive and non-invasive laboratory procedures on rats and mice. Techniques illustrated alongside the text would undoubtedly be useful to both junior and senior molecular biologists. In particular, techniques shown for blood pressure measurements in rats and mice appear to be clear and could be replicable in any molecular biology laboratory. The chapter on mouse surgical models in cardiovascular research is impressive and would be an excellent help in the planning and writing project grant application. Similarly, other chapters would also be helpful in the same context.

There are several chapters that offer guidance on mathematical and statistical aspects of genomics research, for example, quantitative trait loci (QTLs), computing in genetic imprinting, haplotype association mapping, candidate gene association and linkage analysis, bioinformatics analysis of microarray data, expression QTL analysis (eQTLs) in humans and finally analyzing the phenotypic variation with different candidate genes. All these chapters offer a simplified version of the most complicated subjects in genetics and genomics research.

One would agree with the editor that most chapters offer information and practical guidelines in developing individually tailored protocols to enable researchers to identify causal genes and novel molecular targets leading to new therapeutic avenues for cardiovascular disease. However, all these methods are not limited to one system diseases but can be applied to investigate any other body system or organ.

Although this book is well produced with excellent layout, it will probably find a very limited place on any researcher's book shelf. However, most molecular biology laboratories may consider keeping a copy in the library for quick reference and useful in teaching and training of young investigators in molecular genomics.
D. Kumar $(\bowtie)$

Institute of Medical Genetics, University Hospital of Wales, Cardiff University, Cardiff, Wales, UK

e-mail: kumard1@Cardiff.ac.uk 